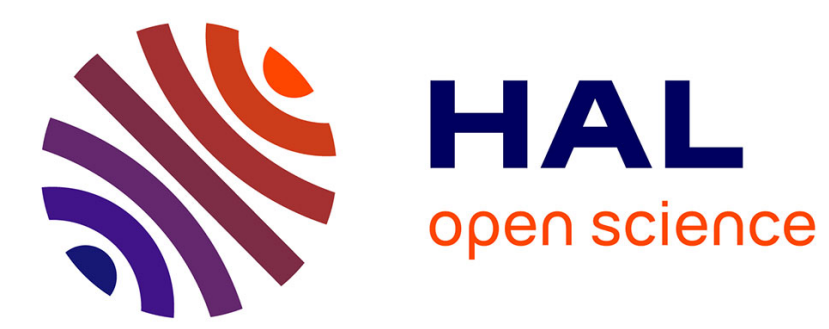

\title{
Real time identification of delay systems
}

\author{
Lotfi Belkoura, Jean-Pierre Richard, Michel Fliess
}

\section{To cite this version:}

Lotfi Belkoura, Jean-Pierre Richard, Michel Fliess. Real time identification of delay systems. Ifac Worshop on Time delay Systems, 2007, Nantes, France. inria-00159773

\section{HAL Id: inria-00159773 https://hal.inria.fr/inria-00159773}

Submitted on 4 Jul 2007

HAL is a multi-disciplinary open access archive for the deposit and dissemination of scientific research documents, whether they are published or not. The documents may come from teaching and research institutions in France or abroad, or from public or private research centers.
L'archive ouverte pluridisciplinaire HAL, est destinée au dépôt et à la diffusion de documents scientifiques de niveau recherche, publiés ou non, émanant des établissements d'enseignement et de recherche français ou étrangers, des laboratoires publics ou privés. 


\title{
REAL TIME IDENTIFICATION OF DELAY SYSTEMS
}

\author{
Lotfi Belkoura*, Jean-Pierre Richard ${ }^{* *}$ \\ and Michel Fliess ${ }^{* * *}$
}

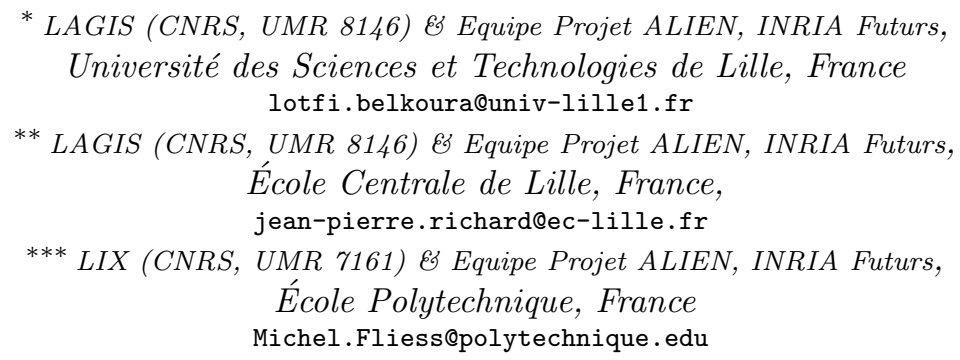

\begin{abstract}
This paper deals with real time and possibly closed loop identification of delay systems. It is based on non-asymptotic algebraic estimation techniques initiated in (Fliess M., 2003). Numerical simulations with noisy data are provided.
\end{abstract}

Keywords: Delay systems, Closed loop Identification.

\section{INTRODUCTION}

The real time delay identification is one of the most crucial open problems in the field of delay systems (see, e.g., (Richard, 2003)). On the one hand, various powerful control techniques (predictors, flatness-based predictive control, finite spectrum assignments, observers, ...) may be applied if the dead-time is known. On the other hand, most existing identification techniques for time-delay systems (see, e.g., (Orlov et al., 2006; Drakunov et al., 2006) for adaptive techniques or (Ren et al., 2005) for a modified least squares technique) generally suffer from poor speed performance. Recent developments in (Belkoura and Richard, 2006; Belkoura et al., 2006) have considered the on line identification of delay systems with particular (finite dimensional) initial conditions but simple simulations easily show that one can not disregard the effects of nonzero (infinite dimensional) initial condition. This paper considers the general and practical corresponding case for which the measurements are not assumed to start with the ex- periment, but may run from an arbitrary starting point. Although the parameter estimation technique is still inspired from the fast identification techniques that were proposed (Fliess M., 2003) for linear, finite-dimensional models, this paper considers a new approach to deal with the delay estimation. Let us recall that those techniques are not asymptotic, and do not need statistical knowledge of the noises corrupting the data. ${ }^{1}$

The approach used in this paper is mainly based on well known facts about the convolution product. Particularly, a delayed signal can be formed from the convolution product $u(t-\tau)=\delta_{\tau} * u$, with $\delta_{\tau}$ the Dirac measure concentrated at $\{\tau\}$. The complement of the largest open subset in which $u$ vanishes is called the support of $u$ and will be denoted $\operatorname{supp} u$. The following property allows

\footnotetext{
1 See, e.g., (Fliess et al., 2005) for linear and nonlinear diagnosis, and (Fliess et al., 2003) for signal processing. Several successful laboratory experiments have already been performed; see, e.g., (Beltran-Carvajal et al., 2005).
} 
local considerations, $\operatorname{supp} u * v \subset \operatorname{supp} u+\operatorname{supp} v$, where the sum in the right hand side is defined by $\{x+y ; x \in \operatorname{supp} u, y \in \operatorname{supp} v\}$. Finally, with no danger of confusion, we shall sometimes denote $u(s), s \in \mathbb{C}$, the Laplace transform of $u$. The paper is organized as follows. Section 2 focuses on a pure delay identification, while Section 3 shows how, combined with the algebraic afore mentioned method, both parameters and delay can be identified for dynamic systems.

\section{IDENTIFICATION PRINCIPLE FOR A SINGLE DELAY}

When considered on the whole real line, a delay between an input $u$ and an output $y$ reads $y(t)=$ $u(t-\tau)$, rewritten as in (1) in a convolution framework, and leading to (2) once multiplied by $(t-\tau)$.

$$
\begin{aligned}
y & =\delta_{\tau} * u, \\
(t-\tau) y & =\delta_{\tau} * t u
\end{aligned}
$$

A convolution product derived from these two relations results in equation (3) without any deviated argument, and from which an explicit and non asymptotic delay formulation is obtained:

$$
\begin{aligned}
& (t-\tau) y * u=t u * y, \\
\Rightarrow & \tau=\frac{t y * u-y * t u}{u * y} .
\end{aligned}
$$

Provided the involved convolution products are well defined, this delay formula holds for all nonzero values of $(u * y)(t)$. Unfortunately, and even in the asymptotic case, this formula no longer holds if $y$ and $u$ are replaced by their restriction to some arbitrary interval $\left(t_{0}, \infty\right)$. More precisely, and as depicted in Figure 1, when restricted to $\left(t_{0}, \infty\right)$, the relationship between $u$ and $y$ involves an additional term $y_{0}$ (initial condition) and should be written:

$$
\begin{aligned}
& y=\delta_{\tau} * u+y_{0}, \\
& \text { with } \quad \operatorname{supp} y_{0} \subset\left(t_{0}, t_{0}+\tau\right) .
\end{aligned}
$$

Since its support is by definition unknown, $y_{0}$ is an unknown signal and some simple simulations have clearly shown that, unless particular cases with zero initial conditions considered in (Belkoura and Richard, 2006), one can not disregard the effects of this term. Due to the translational invariance, we shall restrict our study to $(0, \infty)$.

The main object of the remaining part of this section is the delay estimation based on the only measurement $y$ and $u$ on $(0, \infty)$. In order to cancel

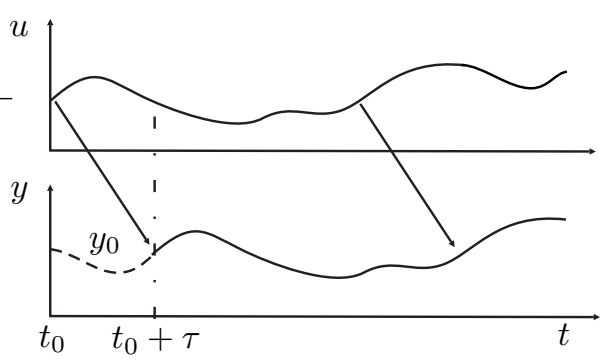

Fig. 1. Measurements and initial condition

the contribution of the initial term $y_{0}$, multiplications by other functions than the simple term $(t-$ $\tau$ ) considered in (2) will be used. More precisely, multiplications of (5) by a set $\left\{\alpha_{n}, n \geq n_{0}\right\}$ of "almost-zero" functions on $(-\tau, 0)$ are considered in order to annihilate the contribution of the initial condition term (i.e. $\alpha_{n}(t-\tau) y_{0} \approx 0, \quad n \geq$ $\left.n_{0}\right)$, yielding from (5):

$$
\alpha_{n}(t-\tau) y \approx \delta_{\tau} * \alpha_{n} u
$$

Hence, considered for $n_{1}, n_{2} \geq n_{0}$, an analog process to $(1,2,3)$ leads to the approximation:

$$
\alpha_{n_{2}}(t-\tau) y * \alpha_{n_{1}} u \approx \alpha_{n_{1}}(t-\tau) y * \alpha_{n_{2}} u
$$

The delay being assumed unknown, the $\alpha_{n}$ functions are necessarily $\tau$ dependent and should satisfy:

$$
\begin{cases}\alpha_{n}(t-\tau) \approx 0, & t \in(0, \tau) \\ \alpha_{n}(t-\tau) \neq 0, & t \notin(0, \tau)\end{cases}
$$

The design of such polynomials comes from the following statement, straightforwardly derived from standard mathematical books (see, e.g., (Sibony and Mardon, 1984)):

Lemma 1. The polynomial of order $n$ that provides the best uniform approximation of the zero function on $[-\tau, 0]$ is given by:

$$
\alpha_{n}(t)=2(-\tau / 4)^{n} T_{n}\left(\frac{2 t}{\tau}+1\right)
$$

where $T_{n}$ is the Tchebytchev polynomial (of the second kind) defined on $[-1,1]$.

More precisely, with $(\sigma, \varepsilon)=(0,1)$ or $(1,-1), C_{k}^{i}$ the binomial coefficients and $\gamma_{k}^{n}$ the coefficients of $T_{n}$, one gets:

$$
\begin{gathered}
\alpha_{n}(t-\sigma \tau)=\sum_{i=0}^{n} \nu_{i}^{n}(\varepsilon) t^{n-i} \tau^{i} \\
\nu_{i}^{n}(\varepsilon)=\left(2^{n-i+1} / 4^{n}\right) \sum_{k-i}^{n} \gamma_{k}^{n} C_{n-i}^{k} \varepsilon^{k+i}
\end{gathered}
$$

Introduced in (7), an after reordering terms, we obtain the following polynomial in $\tau$ and in which, for ease of notations, we have denoted $\nu_{i}^{n}=\nu_{i}^{n}(1)$ and $\bar{\nu}_{i}^{n}=\nu_{i}^{n}(-1)$ : 


$$
\sum_{i=0}^{n_{1}+n_{2}} \xi_{i}(t, y, u) \tau^{i} \approx 0
$$

where

$$
\begin{aligned}
\xi_{i}(t, y, u)= & \sum_{p+q=i} \bar{\nu}_{p}^{n_{2}} \nu_{q}^{n_{1}}\left(t^{n_{2}-p} y * t^{n_{1}-q} u\right) \\
& -\bar{\nu}_{p}^{n_{1}} \nu_{q}^{n_{2}}\left(t^{n_{1}-p} y * t^{n_{2}-q} u\right)
\end{aligned}
$$

Therefore, we have obtained that, for each time $t$, the delay estimation problem has been reduced to the roots computation of the polynomial (12) of order $n_{1}+n_{2}$ and with available coefficients.

The choice of the appropriate root will be discussed in the next section, but we can already note that, for obvious causality reasons, only those satisfying $0<\tau_{i}<t$ will be considered. It should be also noted that, although this is an on-line and non asymptotic technique, relation (12) remains an approximation that depends on the candidate functions $\alpha_{n}$. Note finally that from (13), one can easily show that considering a gain (using for instance $k u$ instead of $u$ ) does not modify the estimation algorithm.

\section{3. (TOWARD A) CLOSED LOOP PARAMETERS AND DELAY IDENTIFICATION}

\subsection{Taking dynamics into account}

In addition to delay estimation, multiplication by polynomials allows simple methods for the identification of linear delay systems. Indeed, integration by parts can be used to deal with derivatives, as it has been used in the non asymptotic methods cited in the introductory section. In our case, and for a first order derivative, the integration by parts decomposition can be written as:

$$
\begin{aligned}
& \xi_{i}(t, \dot{y}, u)=\dot{\xi}_{i}(t, y, u) \\
& -\sum_{\substack{p+q=i \\
p<n_{2}}} \bar{\nu}_{p}^{n_{2}} \nu_{q}^{n_{1}}\left(n_{2}-p\right)\left(t^{n_{2}-p-1} y * t^{n_{1}-q} u\right) \\
& -\sum_{\substack{p+q=i \\
p<n_{1}}} \bar{\nu}_{p}^{n_{1}} \nu_{q}^{n_{2}}\left(n_{1}-p\right)\left(t^{n_{1}-p-1} y * t^{n_{2}-q} u\right) .
\end{aligned}
$$

With such decomposition, and using an additional integration, a delay estimation for first order dynamic systems will be reduced to the single delay estimation of the previous section. The following example illustrates this point.

\subsection{Application to the delay estimation of a closed loop integrator}

In this case study, we consider a closed loop delayed integrator described by:

$$
\frac{y(s)}{\varepsilon(s)}=\frac{k \mathrm{e}^{-\tau s}}{s}, \quad \varepsilon=u-y .
$$

Written in the time domain and taking into account the initial condition $y_{0}$, the input-output relation reads:

$$
\dot{y}=y_{0}+k(u(t-\tau)-y(t-\tau)) .
$$

Note that unlike the previous single delay case, the initial condition term involves a measure and not only a function, since it can be shown that in the distribution sense, $y_{0}=y(0) \delta+k(u(\theta)-y(\theta))$, $-\tau \leq \theta \leq 0$. This remark, however, do not affect the approach, and provided the substitutions in (12):

$$
y \longleftarrow \dot{y} \text { and } u \longleftarrow u-y
$$

we recover the single delay context of Section 2 . The causality in ensured by means of an additional integration, leading to:

$$
\sum_{i=0}^{n_{1}+n_{2}}\left[\int_{0}^{t} \xi_{i}(t, \dot{y}, u-y)\right] \tau^{i} \approx 0 .
$$

The above integrals are performed according to the decomposition provided in (14). Finally, the choice of the appropriate root is based on the stationarity of the delay. More precisely, the selected roots is also assumed to satisfy the auxiliary polynomial $\sum\left[\iint \xi_{i}(t, \dot{y}, u-y)\right] \tau^{i}=0$.

Figure 2 shows simulations and resolution of (15) and (18) in both noise free and noisy contexts, and on a fixed interval (2-10)s.. The polynomial approximations are of order $\left(n_{1}, n_{2}\right)=(5,6)$. The process is described by $k=1, \tau=0.6$, with input $u(t)=.8+\cos 2 t .(0.2+\sin (0.7 t))$. This figure
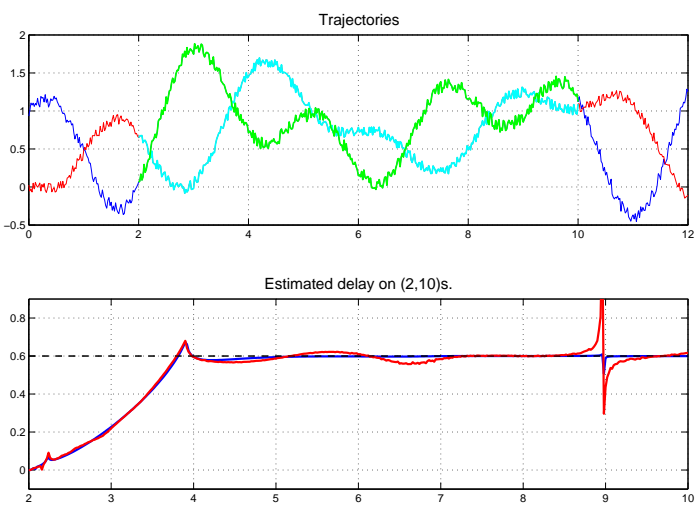

Fig. 2. Estimated delay on section (2-10)s. of the trajectories

clearly shows the convergence of the estimation algorithm to the desired value. The noisy case reveals a singularity at $t=9 \mathrm{~s}$. This is mainly due 
to the particular trajectories on this time interval and can be shifted using different filters instead of the single integration in (18). However, although favorable robustness properties against noise are shown, the control of the singularities remains an open problem and is under investigation.

Remark 2. As in the finite dimensional case (see, e.g., (Fliess and Sira-Ramirez, 2007)), the input signal $u$ being used in this algebraic approach does not necessarily exhibit the classical "persistency of excitation" requirement. However, and although only non trivial trajectories are required, the polynomial coefficients (and hence the roots location) clearly depends on the input-output trajectories. For open loop structures, a constructive method for the design of sufficiently rich inputs for delay systems has been considered in (Belkoura, 2005).

\subsection{Simultaneous closed loop parameters and delay estimation}

The aim of this paragraph is to show how simultaneous parameters and delay estimation can be performed using the same approach. Although the generalization to multiple parameters is straightforward, this example is restricted to a first order system described by:

$$
\frac{y(s)}{\varepsilon(s)}=\frac{k \mathrm{e}^{-\tau s}}{1+a s}, \quad \varepsilon=u-y .
$$

It is readily seen that the following substitutions in (12) may be considered,

$$
y \longleftarrow y+a \dot{y} \text { and } u \longleftarrow u-y,
$$

leading to, by virtue of the linearity of $\xi_{i}$ w.r.t. inputs-outputs,

$$
\sum_{i=0}^{n_{1}+n_{2}}\left[\xi_{i}(t, y, u-y)+a \xi_{i}(t, \dot{y}, u-y)\right] \tau^{i} \approx 0
$$

The estimation of both parameter and delay requires two successive integrations, yielding:

$$
\begin{aligned}
& \sum_{i=0}^{n_{1}+n_{2}}\left[A_{i} \tau^{i}\right] \Theta=0 \quad \text { with } \quad \Theta=\left(\begin{array}{l}
1 \\
a
\end{array}\right) \quad(22) \\
A_{i}= & \left(\begin{array}{cc}
\int \xi_{i}(t, y, u-y) & \int_{i} \xi_{i}(t, \dot{y}, u-y) \\
\int^{(2)} \xi_{i}(t, y, u-y) & \int^{(2)} \xi_{i}(t, \dot{y}, u-y)
\end{array}\right)
\end{aligned}
$$

A generalized eigenvalue problem is hence formed in which the unknown delay consists in one eigenvalue, while the unknown parameters is contained in the corresponding normalized eigenvector. As in the previous case, the appropriate eigenpair is selected from a comparison with an auxiliary eigenvalue problem based on $\int^{t} A_{i}$ instead of $A_{i}$.
Figure 3 shows simulations results with the parameters of the previous section and $a=1.53$. Although the plant is clearly identified, these simulations turned out to be more noise sensitive.

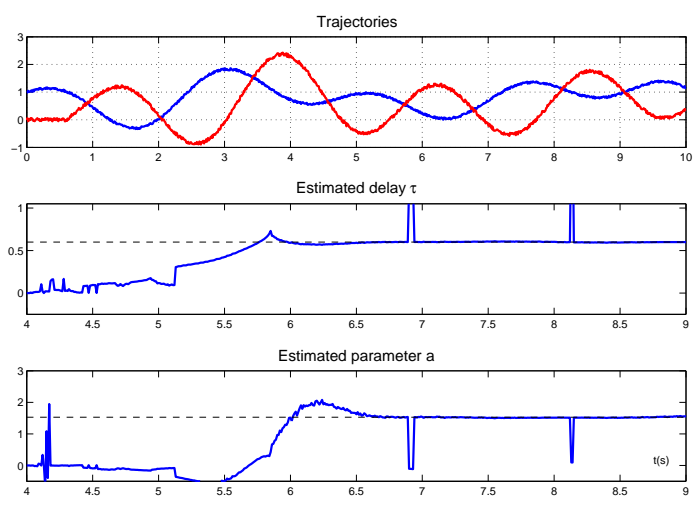

Fig. 3. Estimated delay and parameter on the section (4-9)s. of the trajectories

As in the previous paragraph, the degree of freedom consisting in the choice of the filters involved in (22) remains to be explorated.

\section{CONCLUSION}

This note has presented a new method for closed loop identification of delay systems. The ability of identification of both parameters and delay on a bounded time interval allows to consider for further works the identification of systems with slowly time varying parameters and delay. Extensions to multivariable and multidelay cases, a well as a deeper study of the singularities are also under active investigation.

\section{REFERENCES}

Belkoura, L. (2005). Identifiability of systems described by convolution equations. Automatica 41, 505-512.

Belkoura, L. and J.-P. Richard (2006). A distribution framework for the fast identification of linear systems with delays. 6th IFAC Workshop on Time Delay Systems, TDS06, L Aquila, Italy, July 10-12.

Belkoura, L., J.-P. Richard and M. Fliess (2006). On-line identification of systems with delayed inputs. 16th Conf. Mathematical Theory of Networks 85 Systems, MTNS 06, Kyoto, Japan, July.

Beltran-Carvajal, F., G. Silva-Navarro, H. SiraRamirez and J. Quezada-Andrade (2005). Active vibration control using on-line algebraic identification of harmonic vibrations. Proc. Amer. Control Conf, Portland, OR. 
Drakunov, S.V., W. Perruquetti, J.-P. Richard and L. Belkoura (2006). Delay identification in time-delay systems using variable structure observers. Annual Reviews in Control pp. $143-158$.

Fliess, M. and H. Sira-Ramirez (2007). Closedloop parametric identification for continuoustime linear systems via new algebraic techniques. In: Continuous-Time Model Identifcation from Sampled Data (H. Garnier \& L. Wang, Ed.). Springer, http: //hal.inria.fr/ inria-00114958.

Fliess, M., C. Join and H. Sira-Ramirez (2005). Closed-loop fault-tolerant control for uncertain nonlinear systems. Control and Observer Design for Nonlinear Finite and Infinite Dimensional Systems T.Meurer, K. Graichen, E.D. Gilles (Eds), Lect. Notes Control Informat. Sci., Springer,Berlin 322, 217-233.

Fliess, M., M. Mboup, H. Mounier and H. SiraRamirez (2003). Questioning some paradigms of signal processing via concrete examples. Algebraic Methods in Flatness, Signal Processing and State Estimation, Editiorial Lagares, Mexico pp. 1-21.

Fliess M., Sira-Ramirez H. (2003). An algebraic framework for linear identification. ESAIM Control, Optimization ans Calculus of Variations.

Orlov, Y., I.V. Kolmanovsky and O. Gome (2006). On-line identification of siso linear time-delay systems from output measurements: Theory and applications to engine transient fuel identification. Proceedings of the 2006 American Control Conference Minneapolis, Minnesota, USA, June 14-16.

Ren, X.M., A.B. Rad, P.T. Chan and W.L. Lo (2005). On line identification of continuoustime systems with unknown time delay. Ieee Tac 50-9, 1418-1422.

Richard, JP. (2003). Time-delay systems: an overview of some recent advances and open problems. Automatica 39, 1667-1694.

Sibony, M and J.-C. Mardon (1984). Analyse numérique II. Hermann. Paris. 